\title{
An Interference Alignment Algorithm Based on SLNR for MIMO Interfering Broadcast Channel
}

\author{
Pengfei-Wang ${ }^{1, a, *}$, Shilei-Zhu ${ }^{2, b}, B^{\text {Bin-Ba }}{ }^{1, c}$ \\ ${ }^{1}$ Information Systems Engineering, Information Engineering University, Science Avenue 62, \\ ZhengZhou, China \\ ${ }^{2} 66393$ Postdoctoral Workstation, Seven East Road, Shijiazhuang, China \\ a1150670039@qq.com, ${ }^{\mathrm{b}}$ zhushilei3620@qq.com, ${ }^{\mathrm{c}}$ xidianbabin@163.com \\ *corresponding author
}

Keywords: IBC, MIMO, interference alignment, SLNR

\begin{abstract}
Aiming at the problem that the existing EUG IA algorithm does not have antinoise performance, this paper proposes an interference alignment algorithm based on SLNR. The algorithm uses a hierarchical design idea. The outer layer first uses the extended grouping strategy to group the cells and users, and solves the outer precoding matrix and the interference suppression matrix to eliminate the inter-cell interference, so as to transform the multi-cell multi-user interference cancellation into single-cell multi-user interference cancellation problem. The inner layer uses the Max-SLNR criterion to iterate to solve the single-cell precoding matrix and the interference suppression matrix to eliminate inter-user interference. The simulation results show that the proposed algorithm has better performance and anti-noise performance than the original algorithm.
\end{abstract}

\section{Introduction}

In recent years, how to make efficient use of wireless spectrum resources to improve the capacity of wireless systems has been a hot topic in academic research. In the existing cellular system, the same frequency multiplexing method (reuse factor of 1) can greatly improve the spectrum utilization rate, but it is easy to cause serious inter-cell interference with the same frequency, and finally become a bottleneck to limit the capacity of the system. So, the "Interference Alignment (IA)" technology was proposed in [1][2], which breaks the traditional understanding of the interference network capacity in the traditional sense, and is considered to be important to enhance the future communication system capacity and spectrum efficiency means.

For the application of interference alignment technology in multi-cell multi-user system, domestic and foreign scholars have done extensive research. In [3], the interference technique is first applied to the cell network, and it is proved that it is feasible to interfere with the alignment technique under the broadcast system or multiple access system. Based on this, in the paper [4], a cascade precoding interference alignment method is proposed. The inner layer precoding is used to eliminate inter-user interference (IUI), and the outer precoding is used to eliminate inter-Cell 
interference, ICI). This algorithm has achieved better results under the two communities, but it will cause greater interference leakage for multi-cell systems, due to the need to select the main interference and residual interference, and the remaining interference is treated as noise. In [5], a two-cell interference alignment algorithm is proposed. By aligning the inter-cell interference of each user in the cell into the same subspace, the transmitter uses the zero forcing algorithm to eliminate inter-cell interference and inter-user interference. On the basis of [5], in [6], an interference alignment algorithm of Extension User Group (EUG) is proposed and is extended to any cell. In [7], a regularized zero-interference alignment algorithm is proposed for the two-cell system, which effectively reduces the complexity of the algorithm, but it is difficult to extend to any number of cells. [8] and [9] proposed an opportunistic interference alignment algorithm under broadcast channel. By choosing to communicate with other users with less interference, it can effectively reduce inter-cell interference, but this method will make some users unable to communicate. [10] tried to solve the precoding matrix by using the iteration of the transmitter and receiver, but it will bring the huge feedback between different base stations and users. [11] proposed a triangular decomposition of the interference alignment algorithm, but only applies to three-cell system, multi-cell system does not apply. In [12] and [13], an interference alignment algorithm based on eigenvalue decomposition and minimum mean square error is proposed, which can be applied to multi-cell systems and achieve better results. However, since only two cells are disturbed at a time alignment, the capacity performance achieved with the literature [6] there is still a certain gap.

Thus, on the basis of [6], this paper proposes a SLNR-based packet IA optimization algorithm. The algorithm uses the hierarchical design method, first in the outer layer to obtain the transceiver matrix to eliminate inter-cell interference, so multi-cell multi-user interference broadcast channel scene into a single-cell multi-user broadcast channel scene. And then use the iterative method based on Max-SLNR to find a single cell transceiver matrix to eliminate inter-user interference. Finally, the performance of the algorithm is verified by simulation.

\section{System model}

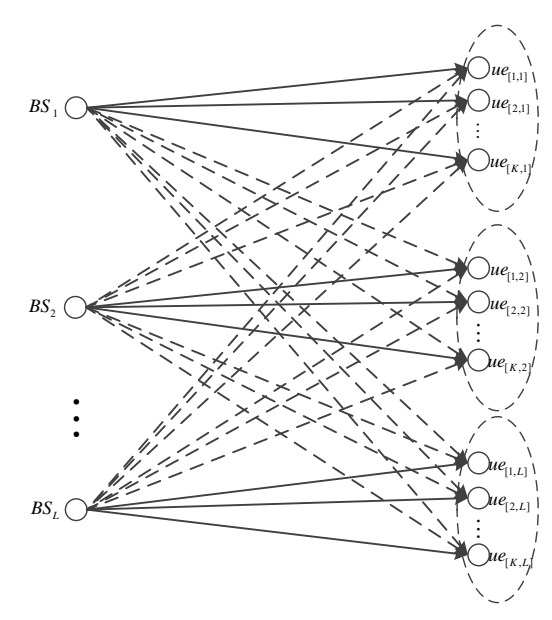

Figure 1 Interference broadcast channel model

In this paper, we consider a multi-cell multi-user interference broadcast channel scenario. There are $L$ cells in the scenario and $K$ users in each cell. The system model is shown in Fig.1. Assuming that each base station node and user node are respectively equipped with the $M$ and $N$ antennas, and $M \geq N$. The power of transmitter is $P$. And the $k$-th user in the $l$-th cell is denoted 
as user $[k, l]$. In the text, $\boldsymbol{I}_{d}$ denotes the $d \times d$ dimensional unit matrix, $(\bullet)^{\mathrm{T}}$ and $(\bullet)^{\mathrm{H}}$ denote the matrix transpose and the matrix conjugate transpose respectively, $\|\bullet\|_{F}^{2}$ denotes the square of the Fnorm of the matrix, and $\operatorname{rank}(\bullet)$ denotes the rank of the matrix. The signal transmitted by the base station $l$ to the user $[k, l]$ can be expressed as

$$
\boldsymbol{x}^{[k, l]}=\boldsymbol{V}^{[k, l]} \boldsymbol{s}^{[k, l]}, k=1,2, \ldots, K ; l=1,2, \ldots, L
$$

where $\boldsymbol{x}^{[k, l]} \in \mathbb{C}^{M \times 1}$ is the transmitted signal matrix of the $k$-th user in the $l$-th cell, which represents the signal transmitted from the antenna after passing through the precoder, $\boldsymbol{s}^{[k, l]}=\left(\boldsymbol{s}_{1}^{[k, l]}, \boldsymbol{s}_{2}^{[k, l]}, \ldots, \boldsymbol{s}_{d_{s}}^{[k, l]}\right)^{\mathrm{T}}$ is the transmitted symbol vector of the $d_{s} \times 1$ dimension, and satisfies an power constraint $E\left(\left\|s^{[k, l]}\right\|_{F}^{2}\right) \leq P . V^{[k, l]}=\left(V_{1}^{[k, l]}, V_{2}^{[k, l]}, \ldots, V_{d_{s}}^{[k, l]}\right)$ is $M \times d_{s}$ dimension precoding matrix, and satisfies $\boldsymbol{V}^{[k, l] \mathrm{H}} \boldsymbol{V}^{[k, l]}=\boldsymbol{I}_{d}$. And $\boldsymbol{V}_{d_{s}}^{[k, l]}$ represents the precoding vector of the $d_{s}$-th data stream of user $[k, l]$. The received signal for user $[k, l]$ can be expressed as

$$
\begin{aligned}
\boldsymbol{Y}^{[k, l]} & =\sum_{i=1}^{L} \boldsymbol{H}_{i}^{[k, l]} \sum_{j=1}^{K} \boldsymbol{x}^{[j, l]}+\boldsymbol{n}^{[k, l]} \\
& =\boldsymbol{H}_{l}^{[k, l]} \boldsymbol{V}^{[k, l]} \boldsymbol{s}^{[k, l]}+\sum_{j=1, j \neq k}^{K} \boldsymbol{H}_{l}^{[k, l]} \boldsymbol{V}^{[j, l]} \boldsymbol{s}^{[j, l]}+\sum_{i=1, i \neq l}^{L} \sum_{j=1}^{K} \boldsymbol{H}_{i}^{[k, l]} \boldsymbol{V}^{[j, i]} \boldsymbol{s}^{[j, i]}+\boldsymbol{n}^{[k, l]}
\end{aligned}
$$

where $\boldsymbol{H}_{l}^{[k, l]} \boldsymbol{V}^{[k, l]} \boldsymbol{s}^{[k, l]}$ denotes the desired signal of the $k$-th user of the $l$-th cell, $\sum_{j=1, j \neq k}^{K} \boldsymbol{H}_{l}^{[k, l]} \boldsymbol{V}^{[j, l]} \boldsymbol{s}^{[j, l]}$ denotes the inter-user interference signal of the user $[k, l]$, $\sum_{i=1, i \neq l}^{L} \sum_{j=1}^{K} \boldsymbol{H}_{i}^{[k, l]} \boldsymbol{V}^{[j, i]} \boldsymbol{s}^{[j, i]}$ denotes the inter-cell interference signal for the user[k,l], $\boldsymbol{n}^{[k, l]}$ denotes the additive white Gaussian noise of the user $[k, l]$, and $\boldsymbol{n}^{[k, l]} \in \mathbb{C}^{N \times 1}$. To suppress the interference and restore the transmit signal, the user $[k, l]$ needs to pass the interference suppression filter. The complete signal processing can be expressed as

$$
\boldsymbol{y}^{[k, l]}=\boldsymbol{U}^{[k, l] \mathrm{H}} \boldsymbol{H}_{l}^{[k, l]} \boldsymbol{V}^{[k, l]} \boldsymbol{S}^{[k, l]}+\boldsymbol{U}^{[k, l] \mathrm{H}}\left(\sum_{j=1, j \neq k}^{K} \boldsymbol{H}_{l}^{[k, l]} \boldsymbol{V}^{[j, l]} \boldsymbol{S}^{[j, l]}+\sum_{i=1, i \neq l}^{L} \sum_{j=1}^{K} \boldsymbol{H}_{i}^{[k, l]} \boldsymbol{V}^{[j, i]} \boldsymbol{S}^{[j, i]}\right)+\tilde{\boldsymbol{n}}^{[k, l]}
$$

where $\boldsymbol{U}^{[k, l]}$ denotes the interference suppression matrix of user $[k, l]$ and $\boldsymbol{U}^{[k, l]} \in \mathbb{C}^{N \times d}$. $\tilde{\boldsymbol{n}}^{[k, l]}=\boldsymbol{U}^{[k, l] \mathrm{H}} \boldsymbol{n}^{[k, l]}$ denotes the additive high white noise signal after passing through the linear filter, which still satisfies the complex Gaussian distribution of $C N(0,1)$. The above is the specific model of the system. 


\section{Extended user group interference alignment}

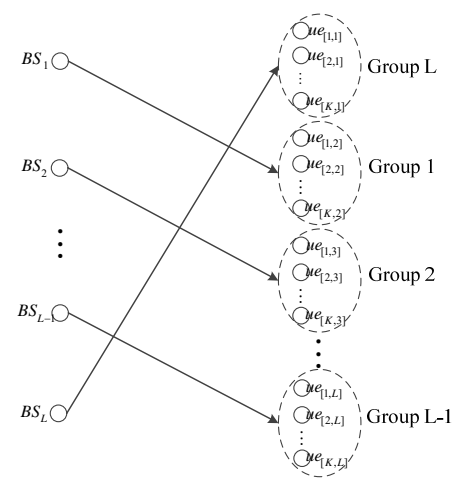

Figure 2 Extended user grouping diagram

As shown in Fig. 2 , the base station $l, l=1,2, \ldots, L-1$ and the users in the cell $l+1$ are grouped into group $l$ according to the paper [5] and [6]. The base station $L$ and the users in the cell 1 are divided into the group $L$. Finally, the entire broadcast interference channel is divided into $L$ groups. Align the interference of the base station within each group to the users:

$$
\boldsymbol{G}_{l}=\operatorname{span}\left(\boldsymbol{H}_{l}^{[1, l+1] \mathrm{H}} \boldsymbol{U}^{[1, l+1]}\right)=\cdots=\operatorname{span}\left(\boldsymbol{H}_{l}^{[k, l+1] \mathrm{H}} \boldsymbol{U}^{[k, l+1]}\right)=\cdots=\operatorname{span}\left(\boldsymbol{H}_{l}^{[K, l+1] \mathrm{H}} \boldsymbol{U}^{[K, l+1]}\right)
$$

Where $l+1=\bmod (l, L)+1, l=1,2, \cdots, L$, and $\operatorname{span}(\boldsymbol{A})$ represents a subspace with a matrix $\boldsymbol{A}$. The solution of this type can be transformed into the form of the following formula,

$$
\left[\begin{array}{ccccc}
\boldsymbol{I}_{M} & -\boldsymbol{H}_{l}^{[1, l+1] \mathrm{H}} & \mathbf{0} & \cdots & \mathbf{0} \\
\boldsymbol{I}_{M} & \mathbf{0} & -\boldsymbol{H}_{l}^{[2, l+1] \mathrm{H}} & \cdots & \mathbf{0} \\
\vdots & \vdots & \vdots & \ddots & \vdots \\
\boldsymbol{I}_{M} & \mathbf{0} & \mathbf{0} & \cdots & -\boldsymbol{H}_{l}^{[K, l+1] \mathrm{H}}
\end{array}\right]\left[\begin{array}{c}
\boldsymbol{G}_{l} \\
\boldsymbol{U}^{[1, l+1]} \\
\boldsymbol{U}^{[2, l+1]} \\
\vdots \\
\boldsymbol{U}^{[K, l+1]}
\end{array}\right]=\overline{\boldsymbol{E}}_{l} \overline{\boldsymbol{U}}_{l}=\mathbf{0}
$$

With this formula, the base station $l$ can be aligned within the same subspace as the inter-cell interference of the user within the cell $l+1$. At the same time, at the base station $l$, the inter-user interference and inter-cell interference of the transmission signal can be eliminated by forcing zero algorithm.

$$
\boldsymbol{V}^{[k, l]} \subset \operatorname{null}\left([\underbrace{\boldsymbol{G}_{l}}_{\text {aligned ICI }} \underbrace{\left(\boldsymbol{U}^{[t(t=1,2, \cdots, K), s(s \neq l, l+1)] \mathrm{H}} \boldsymbol{H}_{i}^{[t(t=1,2, \cdots, K), s(s \neq l, l+1)]}\right)^{\mathrm{H}}}_{\text {unaligned ICI }} \underbrace{\left(\boldsymbol{U}^{[t(t \neq k), l] \mathrm{H}} \boldsymbol{H}_{i}^{[t, l]}\right)^{\mathrm{H}}}_{\text {IUI }}]^{\mathrm{H}}\right)
$$

In the formula (6), the dimension of the extension matrix $\overline{\boldsymbol{E}}_{l}$ is $K M \times(M+K N)$. In order for the equation to be solved and the receiving matrix is at least $d$ dimension, it needs to be satisfied

$$
K M+d \leq(M+K N)
$$

Similarly, for equation (5), all the interference constructed by the matrix dimension is $(L-1) K d \times M$. In order to enable the base station to transmit $d$ non-interference data streams, it needs to be satisfied 


$$
(L-1) K d+d \leq M
$$

The minimum number of transmit and receive antennas can be obtained by combining (7) and (8)

$$
\left\{\begin{array}{c}
M=(L-1) K d+d \\
N=(L-1)(K-1) d+d
\end{array}\right.
$$

\section{The Proposed Iterative SLNR-IA Algorithm}

As mentioned above and as seen from (6), EUG IA algorithm uses the zero forcing algorithm to directly eliminate inter-cell interference and inter-user interference to obtain the precoding matrix, so that the algorithm does not have anti-noise performance. Therefore, this paper proposes a group IA optimization algorithm based on SLNR. And the algorithm uses hierarchical cascading design. The outer layer is designed to eliminate ICI, which take the multi-cell multi-user IBC scene into a traditional single-cell multi-user BC scene. And then uses the Max-SLNR criterion to design the inner layer matrix to eliminate the IUI. Finally, the two layers cascade construct the precoding matrix and the interference suppression matrix. The specific design is as follows.

\subsection{Outer Layer ICI Elimination Design Based on Extended User Grouping}

This paper preserves the idea of extending the group IA in the first step. Firstly, the BS-User is divided into L groups. Then, as in equation (99), the base station $l$ can adjust the ICI of the cell $[l+1]$ into the same subspace. Finally, the corresponding $\boldsymbol{G}_{l} 、 \tilde{\boldsymbol{U}}^{[1, l+1]} 、 \tilde{\boldsymbol{U}}^{[2, l+1]}$ and $\tilde{\boldsymbol{U}}^{[k, l+1]}$ are obtained from the equation (100), where $\tilde{U}^{[k, l+1]}$ represents the outer interference suppression matrix of the $k$-th user of the $[l+1]$ cell. Similarly, the outer interference suppression matrix $\tilde{\boldsymbol{U}}^{[k, l]}$ of all the cells and their users can be obtained, and $l=1,2, \ldots, L, k=1,2, \ldots, K$.

In this paper, $\tilde{\boldsymbol{V}}^{l}$ is defined as the outer precoding matrix of all users of the $l$-th cell, then the matrix should be in the zero space of the other cell receiving matrix, as

$$
\tilde{\boldsymbol{V}}^{l} \subset \operatorname{null}\left(\left[\boldsymbol{G}_{l}\left(\boldsymbol{U}^{[t(t=1,2, \cdots, K), s(s \neq l, l+1)] \mathrm{H}} \boldsymbol{H}_{i}^{[t(t=1,2, \cdots, K), s(s \neq l, l+1)]}\right)^{\mathrm{H}}\right]^{\mathrm{H}}\right)
$$

through the above formula can make all the users within the cell $l$ no interference caused by other cells. At this point, the multi-cell multi-user IBC scene can be transformed into a classic single-cell multi-user $\mathrm{BC}$ scene. The equivalent channel $\hat{\boldsymbol{H}}_{l}^{[k, l]}$ of cell $l$ to user $[k, l]$ can be expressed as

$$
\hat{\boldsymbol{H}}_{l}^{[k, l]}=\tilde{\boldsymbol{U}}^{[k, l] \mathrm{H}} \boldsymbol{H}_{l}^{[k, l]} \tilde{\boldsymbol{V}}^{l}
$$

at this time, the outer precoding matrix and the interference suppression matrix are designed.

\subsection{Inner Layer IUI Elimination Design Based on SLNR}

In [88], the EUG IA algorithm eliminates inter-cell interference and inter-user interference by the formula (101), and finds the precoding matrix of the user[k,l] without considering the antinoise performance of the algorithm. It can not overcome the adverse effects of noise on system performance. Therefore, this paper designs a SLNR criterion after eliminating ICI. By using the generalized Rayleigh quotient, the precoding matrix and the interference suppression matrix of the 
client $[\mathrm{k}, \mathrm{l}]$ are obtained by iterative algorithm. Not only can eliminate the district IUI, but also the algorithm has good anti-noise performance. The specific approach is as follows.

First, the inner layer precoding matrix is defined as $\overline{\boldsymbol{V}}^{[k, l]}$ and the interference suppression matrix is $\overline{\boldsymbol{U}}^{[k, l]}$. The SLNR function for user $[k, l]$ can be expressed as

$$
\operatorname{SLNR} R^{[k, l]}=\frac{\left\|\overline{\boldsymbol{U}}^{[k, l] \mathrm{H}} \hat{\boldsymbol{H}}_{l}^{[k, l]} \overline{\boldsymbol{V}}^{[k, l]}\right\|_{F}^{2}}{\sum_{j=1, j \neq k}^{K}\left\|\overline{\boldsymbol{U}}^{[j, l] \mathrm{H}} \hat{\boldsymbol{H}}_{l}^{[j, l]} \overline{\boldsymbol{V}}^{[k, l]}\right\|_{F}^{2}+\sigma^{2}}
$$

Therefore, the optimal objective function for solving is

$$
\begin{aligned}
\max _{\overline{\boldsymbol{V}}^{[k, l]}, \overline{\boldsymbol{U}}^{[k, l]}} & \operatorname{SLNR} R^{[k, l]} \\
= & \max _{\overline{\boldsymbol{V}}^{[k, l]}, \bar{U}^{[k, l]}} \frac{\operatorname{tr}\left(\overline{\boldsymbol{V}}^{[k, l] \mathrm{H}}\left(\hat{\boldsymbol{H}}_{l}^{[k, l] \mathrm{H}} \overline{\boldsymbol{U}}^{[k, l]} \overline{\boldsymbol{U}}^{[k, l] \mathrm{H}} \hat{\boldsymbol{H}}_{l}^{[k, l]}\right) \overline{\boldsymbol{V}}^{[k, l]}\right)}{\operatorname{tr}\left(\overline{\boldsymbol{V}}^{[k, l] \mathrm{H}}\left(\sum_{j=1, j \neq k}^{K} \hat{\boldsymbol{H}}_{l}^{[j, l] \mathrm{H}} \overline{\boldsymbol{U}}^{[j, l]} \overline{\boldsymbol{U}}^{[j, l] \mathrm{H}} \hat{\boldsymbol{H}}_{l}^{[j, l]}+\sigma^{2} I\right) \overline{\boldsymbol{V}}^{[k, l]}\right)}
\end{aligned}
$$

From the equation (13), we can see that it is a multi-objective optimization problem. Therefore, we can fix one of them to solve the other, and iterate over it, finally find the inner precoding matrix $\overline{\boldsymbol{V}}^{[k, l]}$ and interference suppression matrix $\overline{\boldsymbol{U}}^{[k, l]}$ which satisfies the condition. As follows:

1) Known $\overline{\boldsymbol{V}}^{[k, l]}$, solve $\overline{\boldsymbol{U}}^{[k, l]}$

Initializing the matrix $\overline{\boldsymbol{V}}^{[k, l]}$ that satisfies $\overline{\boldsymbol{V}}^{[k, l]} \overline{\boldsymbol{V}}^{[k, l] \mathrm{H}}=\boldsymbol{I}_{d}$. So that the corresponding interference suppression matrix can be obtained ${ }^{[77]}$ :

$$
\overline{\boldsymbol{U}}^{[k, l]}=\frac{\left(\hat{\boldsymbol{H}}_{l}^{[k, l]} \overline{\boldsymbol{V}}^{[k, l]}\right)^{\mathrm{H}}}{\left\|\hat{\boldsymbol{H}}_{l}^{[k, l]} \overline{\boldsymbol{V}}^{[k, l]}\right\|_{F}}
$$

2) Known $\overline{\boldsymbol{U}}^{[k, l]}$, solve $\overline{\boldsymbol{V}}^{[k, l]}$

The solution of the formula (13) is transformed into the generalized Rayleigh quotient form. The optimal precoding matrix is the matrix of the eigenvectors corresponding to the largest $d$ eigenvalues in the generalized eigenvalues of

$$
\begin{aligned}
\left\{\hat{\boldsymbol{H}}_{l}^{[k, l] \mathrm{H}} \overline{\boldsymbol{U}}^{[k, l]} \overline{\boldsymbol{U}}^{[k, l] \mathrm{H}} \hat{\boldsymbol{H}}_{l}^{[k, l]}, \sum_{j=1, j \neq k}^{K} \hat{\boldsymbol{H}}_{l}^{[j, l] \mathrm{H}} \overline{\boldsymbol{U}}^{[j, l]} \overline{\boldsymbol{U}}^{[j, l] \mathrm{H}} \hat{\boldsymbol{H}}_{l}^{[j, l]}+\sigma^{2} I\right\} \text {, as } \\
\overline{\boldsymbol{V}}^{[k, l]}=\text { eigenvector }\left\{\hat{\boldsymbol{H}}_{l}^{[k, l] \mathrm{H}} \overline{\boldsymbol{U}}^{[k, l]} \overline{\boldsymbol{U}}^{[k, l] \mathrm{H}} \hat{\boldsymbol{H}}_{l}^{[k, l]}, \sum_{j=1, j \neq k}^{K} \hat{\boldsymbol{H}}_{l}^{[j, l] \mathrm{H}} \overline{\boldsymbol{U}}^{[j, l]} \overline{\boldsymbol{U}}^{[j, l] \mathrm{H}} \hat{\boldsymbol{H}}_{l}^{[j, l]}+\sigma^{2} \boldsymbol{I}\right\}
\end{aligned}
$$

Finally, the optimized precoding matrix $\boldsymbol{V}^{[k, l]}$ and the interference suppression matrix $\boldsymbol{U}^{[k, l]}$ of the base station $l$ to the user $[k, l]$ are obtained by the cascade of the inner and outer matrices, which can be expressed as

$$
\begin{gathered}
\boldsymbol{V}^{[k, l]}=\tilde{\boldsymbol{V}}^{l} \overline{\boldsymbol{V}}^{[k, l]} \\
\boldsymbol{U}^{[k, l]}=\overline{\boldsymbol{U}}^{[k, l]} \tilde{\boldsymbol{U}}^{[k, l]}
\end{gathered}
$$

Finally, the formula for calculating the capacity of the system is given as follows

where

$$
C=\sum_{l=1}^{L} \sum_{k=1}^{K} \log _{2}\left|\boldsymbol{I}_{d}+\frac{\boldsymbol{U}^{[k, l] \mathrm{H}} \boldsymbol{H}_{l}^{[k, l]} \boldsymbol{V}^{[k, l]} \boldsymbol{V}^{[k, l] \mathrm{H}} \boldsymbol{H}_{l}^{[k, l] \mathrm{H}} \boldsymbol{U}^{[k, l]}}{\boldsymbol{U}^{[k, l] \mathrm{H}}\left(\boldsymbol{Q}^{[k, l]}+\sigma^{2} \boldsymbol{I}_{N}\right) \boldsymbol{U}^{[k, l]}}\right|
$$




$$
\boldsymbol{Q}^{[k, l]}=\sum_{i=1, i \neq l}^{L} \sum_{j=1}^{K} \boldsymbol{H}_{i}^{[k, l]} \boldsymbol{V}^{[j, i]} \boldsymbol{V}^{[j, i] \mathrm{H}} \boldsymbol{H}_{i}^{[k, l] \mathrm{H}}+\sum_{j=1, j \neq k}^{K} \boldsymbol{H}_{l}^{[k, l]} \boldsymbol{V}^{[j, l]} \boldsymbol{V}^{[j, l] \mathrm{H}} \boldsymbol{H}_{l}^{[k, l] \mathrm{H}}
$$

\subsection{Algorithm Steps}

The design steps of this paper are as follows:

\section{The Proposed Iterative SLNR-IA Algorithm}

STEP1: User grouping,

STEP2: Calculate $\boldsymbol{G}_{l}$ and $\tilde{\boldsymbol{U}}^{[k, l]}$ according to formula (5), where $l=1,2, \ldots, L ; k=1,2, \ldots, K$

STEP3: Calculate the outer precoding matrix $\tilde{\boldsymbol{V}}^{l}$ according to (10),

STEP4: Calculate the inner precoding matrix $\overline{\boldsymbol{V}}^{[k, l]}$ and interference suppression matrix $\overline{\boldsymbol{U}}^{[k, l]}$ according to (14)(15),

STEP5: Calculate the optimized precoding matrix $\boldsymbol{V}^{[k, l]}$ and the interference suppression matrix $\boldsymbol{U}^{[k, l]}$ according to (16)(17).

\section{Simulation}

The simulation experiments are carried out and compared with the [6]. Assuming that the system has symmetry, that is, the number of users in each cell is the same, the number of transmitting antennas and the number of receiving antennas are the same, and the number of the respective configurations is set according to the minimum number. And the transmitter has the same performance configuration and transmit power, and the transmit power is normalized to 1 , and the transmission data streams are equally distributed. $(L, K, M, N)$ represents the number of cells configured by the system, the number of users, the number of transmitting antennas, the number of receiving antennas. $d$ is the number of user data streams. And the number of Monte Carlo is 50 .

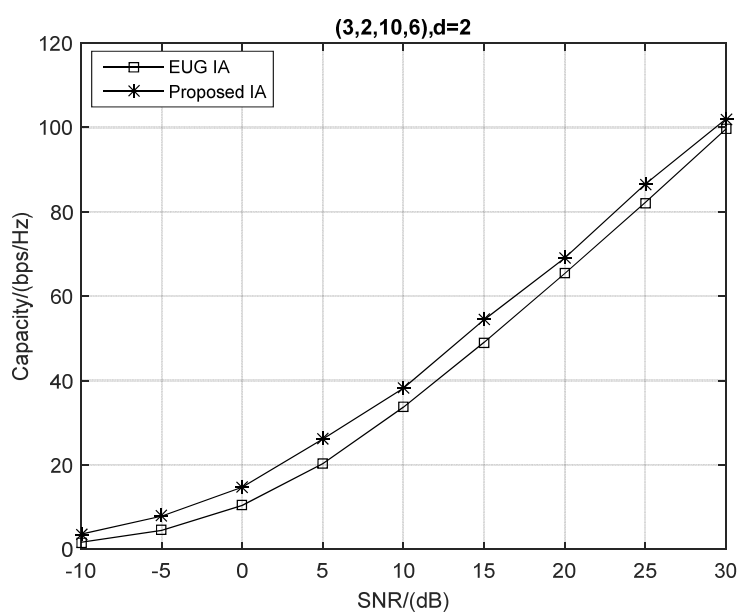

Figure 3 System capacity of $(3,2,10,6)$

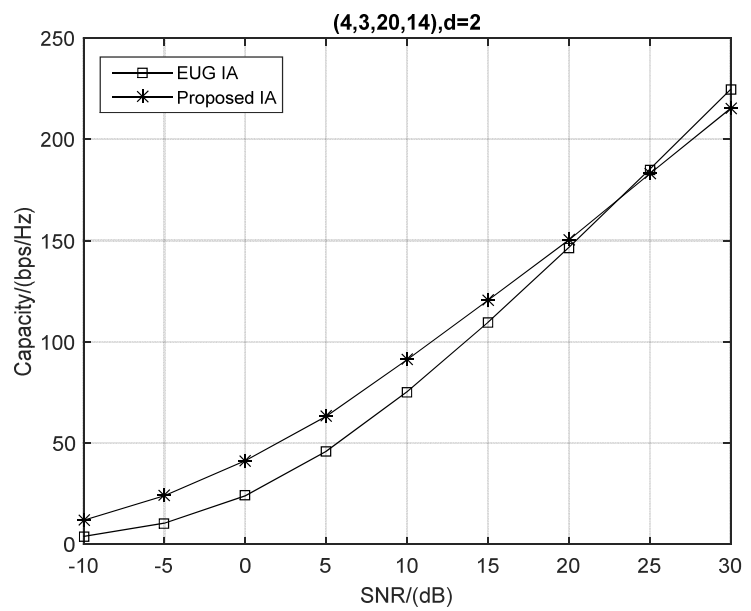

Figure 4 System capacity of $(4,3,20,14)$

Fig. 3 and Fig. 4 show graphs showing the system capacity changes with the signal-to-noise ratio when the system is configured as $(3,2,10,6),(4,3,20,14)$ data streams of 2 , respectively. It can be seen from the figure that the system capacity of the proposed algorithm is better than that of the EUG IA algorithm. This is because the traditional algorithm solves the IUI and ICI by using the zero forcing algorithm while solving the precoding matrix. And the algorithm itself does not consider the influence of noise. In this paper, the proposed algorithm is different from the original 
algorithm while eliminating the ICI and IUI practice. Firstly, we transform the multi-cell multi-user interference broadcast scene into the traditional broadcast channel scene of single-cell multi-user, and then the SLNR algorithm is used to obtain the inner layer Precoding matrix and interference suppression matrix, taking into account the impact of noise, with better system performance.

\section{References}

[1] Cadambe V R, Jafar S A. Interference Alignment and the Degrees of Freedom for the K User Interference Channel[J]. IEEE Transactions on Information Theory, 2007, abs/0707. 0323(8): 3425--3441.

[2] Gomadam K, Cadambe V R, Jafar S A. Approaching the Capacity of Wireless Networks through Distributed Interference Alignment [C]// Proceedings of the GLOBECOM. New York: IEEE, 2008:4260-4265.

[3] Suh C, Tse D. Interference Alignment for Cellular Networks[C]// Communication, Control, and Computing, 2008 46th Annual Allerton Conference on. IEEE, 2008:1037-1044.

[4] Suh C, Ho M, Tse D N C. Downlink Interference Alignment[C]// Conference on Circuits, Systems \& Computers. 2010:2616-2626.

[5] Shin W, Lee N, Lim J B, et al. On the Design of Interference Alignment Scheme for Two-Cell MIMO Interfering Broadcast Channels[J]. IEEE Transactions on Wireless Communications, 2011, 10(2):437-442.

[6] Tang J, Lambotharan S. Interference Alignment Techniques for MIMO Multi-Cell Interfering Broadcast Channels[J]. IEEE Transactions on Communications, 2013, 61(1):164-175.

[7] Shin J, Moon J. Regularized Zero-Forcing Interference Alignment for the Two-Cell MIMO Interfering Broadcast Channel[J]. Communications Letters IEEE, 2013, 17(7):1336-1339.

[8] Yang H J, Shin W Y, Bang C J, et al. Opportunistic interference alignment for MIMO interfering broadcast channels[C]// Acoustics, Speech and Signal Processing (ICASSP), 2014 IEEE International Conference on. IEEE, 2014:2425-2429.

[9] Yang H J, Shin W Y, Bang C J, et al. Opportunistic downlink interference alignment[C]// Information Theory (ISIT), 2014 IEEE International Symposium on. IEEE, 2014:1588-1592.

[10] Schreck J, Wunder G. Iterative interference alignment for cellular systems with user selection[C]// Acoustics, Speech and Signal Processing (ICASSP), 2012 IEEE International Conference on. IEEE, 2012:2833-2836.

[11] Xie X Z, Xu B, Lei W J, et al. Interference Alignment Algorithm Based on Orthogonal-triangular Decomposition for Edge Users in 3-cell MIMO Cellular Networks[J]. Dianzi Yu Xinxi Xuebao/journal of Electronics \& Information Technology, 2013, 35(5):1031-1036.

[12] JIAO Xiao-rong, ZHAO Yue-fang, ZHANG Zu-fan, et al. Interference Alignment Algorithm Based on Eigenvector Splitting in MIMO-IBC [J]. Huazhong University of Science and Technology: Natural Science Edition, 2015, 43 (8): 70-74.

[13] Shi-Dang L I, Chun-Guo L I, Wang Y, et al. A Transceiver Design Algorithm of Weighted Mean Square Error Minimization for the Multicell Broadcast Interfering Channel[J]. Journal of Signal Processing, 2015. 